UT-678

April '94

\title{
Generalized Pauli-Villars Regularization and the Covariant Form of Anomalies
}

\author{
Kazuo Fujikawa \\ Department of Physics, University of Tokyo \\ Bunkyo-ku,Tokyo 113, Japan
}

\begin{abstract}
In the generalized Pauli-Villars regularization of chiral gauge theory proposed by Frolov and Slavnov, it is important to specify how to sum the contributions from an infinite number of regulator fields. It is shown that an explicit sum of contributions from an infinite number of fields in anomaly-free gauge theory essentially results in a specific choice of regulator in the past formulation of covariant anomalies. We show this correspondence by reformulating the generalized Pauli- Villars regularization as a regularization of composite current operators. We thus naturally understand why the covariant fermion number anomaly in the Weinberg-Salam theory is reproduced in the generalized Pauli-Villars regularization. A salient feature of the covariant regularization, which is not implemented in the lagrangian level in general but works for any chiral theory and gives rise to covariant anomalies, is that it spoils the Bose symmetry in anomalous theory. The covariant regularization however preserves the Bose symmetry as well as gauge invariance in anomaly-free gauge theory.
\end{abstract}




\section{Introduction}

An interesting regularization of chiral gauge theory in the Lagrangian level has been proposed by Frolov and Slavnov [1]. This scheme incorporates an infinite number of bosonic and fermionic regulator fields, and as such how to sum the contributions from the infinite number of regulator fields constitutes an essential part of this regularization ; a formal introduction of an infinite number of regulator fields in the Lagrangian does not completely specify the theory. Detailed analysis of this regularization scheme have been also performed by several authors [2] [3] : For example, the covariant form of anomaly for the fermion number current in the Weinberg-Salam theory $\llbracket$ is naturally reproduced in the generalized Pauli-Villars regularization[3].

The purpose of the present paper is to analyze the basic mechanism of this regularization scheme from a general view point of regularization and anomalies [5]. We show that the generalized Pauli-Villars regularization, after one sums the contributions from an infinite number of fields, essentially corresponds to a specific choice of regulator in the calculational scheme of covariant anomalies [6] [7]. The covariant form of fermion number anomaly is thus naturally understood. A reformulation of the genaralized Pauli-Villars regularization as a regularization of composite current operators is crucial in this analysis. The calculational scheme of covariant anomalies, which works for any chiral gauge theory,was introduced as a convenient means in the path integral formulation of anomalous identities [6] [8]. This regularization is not implemented in the Lagrangian level,but rather it regularizes various currents and amplitudes directly. In terms of Feynman diagrams , this regularization imposes the gauge invariance on all the vertices except for the one corresponding to the Noether current generated by the change of path integral variables. The anomaly produced at the Noether current is thus "gauge covariant" , but it explicitly spoils the Bose symmetry of the underlying Feynman diagrams. If one applies this regularization to non-anomalous diagrams, however, the Noether current is conserved and the Bose symmetry in Feynman diagrams is preserved.

In the following, we show the above correspondence ( and also some difference ) between the generalized Pauli-Villars regularization and the calculational scheme of covariant anomalies by explicitly evaluating several anomalous as well as non-anomalous 
diagrams.

\section{Generalized Pauli-Villars Regularization}

We first recapitulate the essence of the generalized Pauli-Villars regularization and reformulate it as a regularization of composite current operators. The starting theory which we want to regularize is defined by

$$
\mathcal{L}=\bar{\psi} i \not D\left(\frac{1+\gamma_{5}}{2}\right) \psi
$$

where

$$
\begin{aligned}
\not D & =\gamma^{\mu}\left(\partial_{\mu}-i g A_{\mu}^{a}(x) T^{a}\right) \\
& \equiv \gamma^{\mu}\left(\partial_{\mu}-i g A_{\mu}(x)\right)
\end{aligned}
$$

and $T^{a}$ is the hermitian generator of a compact semi-simple group,

$$
\left[T^{a}, T^{b}\right]=i f^{a b c} T^{c}, \quad \operatorname{Tr} T^{a} T^{b}=\frac{1}{2} \delta^{a b} .
$$

In the main part of this paper, we treat the gauge field $A_{\mu}(x)$ as a background field,and the dynamical aspects of $A_{\mu}$ will be briefly commented on later. In the Euclidean metric we use, the $\gamma$ - matrices satisfy

$$
\begin{gathered}
\left\{\gamma^{\mu}, \gamma^{\nu}\right\}=2 g^{\mu \nu} \quad, \quad g^{\mu \nu}=(-1,-1,-1,-1) \\
\left(\gamma^{\mu}\right)^{\dagger}=-\gamma^{\mu} \quad, \quad \gamma_{5}^{\dagger}=\gamma_{5} \\
\left(\gamma_{5}\right)^{2}=1 .
\end{gathered}
$$

The Dirac operator $\not D$ is formally hermitian for the natural inner product of Euclidean theory

$$
\begin{aligned}
(\Phi, \not D \Psi) & \equiv \int d^{4} x \Phi^{\dagger} \not D \Psi \\
& =(\not D \Phi, \Psi) .
\end{aligned}
$$

The generalized Pauli-Villars regularization of $(2,1)$ is defined by 


$$
\begin{aligned}
\mathcal{L}= & \bar{\psi} i \not D \psi-\bar{\psi}_{L} M \psi_{R}-\bar{\psi}_{R} M^{\dagger} \psi_{L} \\
& +\bar{\phi} i \not \supset \phi-\bar{\phi} M^{\prime} \phi
\end{aligned}
$$

where

$$
\psi_{R}=\frac{1}{2}\left(1+\gamma_{5}\right) \psi \quad, \quad \psi_{L}=\frac{1}{2}\left(1-\gamma_{5}\right) \psi
$$

and the infinite dimensional mass matrices in (2.5) are defined by

$$
\begin{aligned}
& M=\left(\begin{array}{ccccc}
0 & 2 & 0 & 0 & \cdots \\
0 & 0 & 4 & 0 & \cdots \\
0 & 0 & 0 & 6 & \cdots \\
\cdots & & & &
\end{array}\right) \Lambda \\
& M^{\dagger} M=\left(\begin{array}{ccccc}
0 & & & & \\
& 2^{2} & & 0 & \\
& & 4^{2} & & \\
& 0 & & 6^{2} & \\
& & & & \ddots
\end{array}\right) \Lambda^{2} \\
& M M^{\dagger}=\left(\begin{array}{cccc}
2^{2} & & & \\
& 4^{2} & & 0 \\
& & 6^{2} & \\
& 0 & & \ddots
\end{array}\right) \Lambda^{2} \\
& M^{\prime}=\left(\begin{array}{cccc}
1 & & & \\
& 3 & & 0 \\
& & 5 & \\
& 0 & & \ddots
\end{array}\right) \Lambda=\left(M^{\prime}\right)^{\dagger}
\end{aligned}
$$


where $\Lambda$ is a parameter with dimensions of mass.

The fields $\psi$ and $\phi$ in (2.5) then contain an infinite number of components, each of which is a conventional 4-component Dirac field; $\psi(x)$ consists of conventional anticommuting (Grassmann) fields, and $\phi(x)$ consists of commuting bosonic Dirac fields. The regularization (2.5) corresponds to the so-called "vector - like" formulation [2].

The Lagrangian (2.5) is invariant under the gauge transformation

$$
\begin{aligned}
\psi(x) & \rightarrow \psi^{\prime}(x)=U(x) \psi(x) \equiv \exp \left[i w^{a}(x) T^{a}\right] \psi(x) \\
\bar{\psi}(x) & \rightarrow \bar{\psi}^{\prime}(x)=\bar{\psi}(x) U(x)^{\dagger} \\
\phi(x) & \rightarrow \phi^{\prime}(x)=U(x) \phi(x) \\
\bar{\phi}(x) & \rightarrow \bar{\phi}^{\prime}(x)=\bar{\phi}(x) U(x)^{\dagger} \\
\not D & \rightarrow \not D^{\prime}=U(x) \not D U(x)^{\dagger}
\end{aligned}
$$

The Noether current associated with the gauge coupling in (2.5) is defined by the infinitesimal change of matter variables in $(2.8)$ with $\not D$ kept fixed :

$$
\begin{gathered}
\mathcal{L}^{\prime}=\bar{\psi}^{\prime} i \not D \psi^{\prime}-\bar{\psi}_{L}^{\prime} M \psi_{R}^{\prime}-\bar{\psi}_{R}^{\prime} M^{\dagger} \psi_{L}^{\prime} \\
+\bar{\phi}^{\prime} i \not D \phi^{\prime}-\bar{\phi}^{\prime} M^{\prime} \phi^{\prime} \\
=-\left(D_{\mu} w\right)^{a} J^{\mu a}(x)+\mathcal{L}
\end{gathered}
$$

with

$$
J^{\mu a}(x)=\bar{\psi}(x) T^{a} \gamma^{\mu} \psi(x)+\bar{\phi}(x) T^{a} \gamma^{\mu} \phi(x)
$$

Similarly, the U(1) transformation

$$
\begin{aligned}
& \psi(x) \rightarrow e^{i \alpha(x)} \psi(x), \bar{\psi}(x) \rightarrow \bar{\psi}(x) e^{-i \alpha(x)} \\
& \phi(x) \rightarrow e^{i \alpha(x)} \phi(x), \bar{\phi}(x) \rightarrow \bar{\phi}(x) e^{-i \alpha(x)}
\end{aligned}
$$

gives rise to the $\mathrm{U}(1)$ fermion number current

$$
J^{\mu}(x)=\bar{\psi}(x) \gamma^{\mu} \psi(x)+\bar{\phi}(x) \gamma^{\mu} \phi(x)
$$

The chiral transformation 


$$
\begin{aligned}
& \psi(x) \quad \rightarrow \quad e^{i \alpha(x) \gamma_{5}} \psi(x), \bar{\psi} \rightarrow \bar{\psi}(x) e^{i \alpha(x) \gamma_{5}} \\
& \phi(x) \rightarrow e^{i \alpha(x) \gamma_{5}} \phi(x), \bar{\phi} \rightarrow \bar{\phi}(x) e^{i \alpha(x) \gamma_{5}}
\end{aligned}
$$

gives the $\mathrm{U}(1)$ chiral current

$$
J_{5}^{\mu}(x)=\bar{\psi}(x) \gamma^{\mu} \gamma_{5} \psi(x)+\bar{\phi}(x) \gamma^{\mu} \gamma_{5} \phi(x)
$$

Considering the variation of action under the transformation (2.9) and (2.11), one can show that the vector currents (2.10) and (2.12) are naively conserved 1

$$
\begin{aligned}
\left(D_{\mu} J^{\mu}\right)^{a}(x) & \equiv \partial_{\mu} J^{\mu a}(x)+g f^{a b c} A_{\mu}^{b}(x) J^{\mu c}(x)=0 \\
\partial_{\mu} J^{\mu}(x) & =0
\end{aligned}
$$

whereas the chiral current (2.14) satisfies the naive identity

$$
\partial_{\mu} J_{5}^{\mu}(x)=2 i \bar{\psi}_{L} M \psi_{R}-2 i \bar{\psi}_{R} M^{\dagger} \psi_{L}+2 i \bar{\phi} M^{\prime} \gamma_{5} \phi
$$

The quantum theory of (2.5) may be defined by the path integral as

$$
\begin{aligned}
Z & =\int D \bar{\psi} D \psi D \bar{\phi} D \phi \exp \left[\int \mathcal{L} d^{4} x\right] \\
& \equiv \int d \mu \exp \left[\int \mathcal{L} d^{4} x\right]
\end{aligned}
$$

and , for example ,

$$
<\bar{\psi}(x) T^{a} \gamma^{\mu} \psi(x)>=\int d \mu \bar{\psi}(x) T^{a} \gamma^{\mu} \psi(x) \exp \left[\int \mathcal{L} d^{4} x\right]
$$

The path integral over the bosonic variables $\phi$ and $\bar{\phi}$ for the Dirac operator in Euclidean theory needs to be defined via a suitable rotation in the functional space.

${ }^{*}$ The fact that the regularized currents satisfy anomaly-free relations (2.15) shows that the regularization (2.5) is ineffective for the evaluation of possible anomalies in these vector currents. 


\section{Definition of Currents in Terms of Propagators}

We now define the currents in terms of propagators. The basic idea of this approach is explained for the un-regularized theory in (1) as follows : We start with the current associated with the gauge coupling

$$
\begin{aligned}
< & \bar{\psi}(x) T^{a} \gamma^{\mu}\left(\frac{1+\gamma_{5}}{2}\right) \psi(x)> \\
& =\lim _{y \rightarrow x}<T^{*} \bar{\psi}(y) T^{a} \gamma^{\mu}\left(\frac{1+\gamma_{5}}{2}\right) \psi(x)> \\
& =-\lim _{y \rightarrow x}<T^{*}\left(T^{a}\right)_{b c} \gamma_{\alpha \delta}^{\mu}\left(\frac{1+\gamma_{5}}{2}\right)_{\delta \beta} \psi_{\beta c}(x) \bar{\psi}_{\alpha b}(y)> \\
& =\lim _{y \rightarrow x} \operatorname{Tr}\left[T^{a} \gamma^{\mu}\left(\frac{1+\gamma_{5}}{2}\right) \frac{1}{i \not D} \delta(x-y)\right]
\end{aligned}
$$

where we used the anti-commuting property of $\psi$ and the expression of the propagator

$$
<T^{*} \psi(x) \bar{\psi}(y)>=\left(\frac{1+\gamma_{5}}{2}\right) \frac{(-1)}{i \not D_{x}} \delta(x-y)
$$

The trace in (2.19) runs over the Dirac and Yang-Mills indices. We now notice the expansion

$$
\begin{aligned}
\frac{1}{i \not D}= & \frac{1}{i \not \partial+g \not A} \\
= & \frac{1}{i \not \partial}+\frac{1}{i \not \partial}(-g \not A) \frac{1}{i \not \supset} \\
& +\frac{1}{i \not \partial}(-g \not A) \frac{1}{i \not \partial}(-g \not A) \frac{1}{i \not \partial}+\cdots
\end{aligned}
$$

When one inserts (2.21) into (2.19) and retains only the terms linear in $A_{\nu}^{b}(x)$, one obtains

$$
\begin{aligned}
& \lim _{y \rightarrow x} \operatorname{Tr}[\left.T^{a} \gamma^{\mu}\left(\frac{1+\gamma_{5}}{2}\right) \frac{(-1)}{i \not \partial} \gamma^{\nu} T^{b} g A_{\nu}^{b}(x) \frac{1}{i \not \partial} \delta(x-y)\right] \\
&=\lim _{y \rightarrow x} \int d^{4} z \operatorname{Tr}\left[T^{a} \gamma^{\mu}\left(\frac{1+\gamma_{5}}{2}\right) \frac{(-1)}{i \not \partial}\right. \\
& \times \\
&\left.\times \delta(x-z) T^{b} \gamma^{\nu} \frac{1}{i \not \partial} \delta(x-y)\right] g A_{\nu}^{b}(z)
\end{aligned}
$$

where the derivative $\partial_{\mu}$ acts on all the $\mathrm{x}$ - variables standing on the right of it in (2.22). If one takes the variational derivative of $(2.22)$ with respect to $g A_{\nu}^{b}(z)$, one obtains 


$$
\begin{aligned}
& \lim _{y \rightarrow x} \operatorname{Tr}\left[T^{a} \gamma^{\mu}\left(\frac{1+\gamma_{5}}{2}\right) \frac{(-1)}{i \not \partial} \delta(x-z) \gamma^{\nu} T^{b} \frac{1}{i \not \partial} \delta(x-y)\right] \\
& \quad=\lim _{y \rightarrow x} \int \frac{d^{4} q}{(2 \pi)^{4}} \frac{d^{4} k}{(2 \pi)^{4}} \operatorname{Tr}\left[T^{a} \gamma^{\mu}\left(\frac{1+\gamma_{5}}{2}\right) \frac{(-1)}{\not k+\not 1} T^{b} \gamma^{\nu} \frac{1}{\not k}\right] e^{-i q(x-z)} e^{-i k(x-y)} \\
& \quad=\int \frac{d^{4} q}{(2 \pi)^{4}} e^{-i q(x-z)}(-1) \int \frac{d^{4} k}{(2 \pi)^{4}} \operatorname{Tr}\left[T^{a} \gamma^{\mu}\left(\frac{1+\gamma_{5}}{2}\right) \frac{1}{\not k+\not q^{2}} T^{\nu}\left(\frac{1+\gamma_{5}}{2}\right) \frac{1}{\not k}\right] \\
& \quad \equiv \int \frac{d^{4} q}{(2 \pi)^{4}} e^{-i q(x-z)} \Pi_{\mu \nu}^{a b}(q)
\end{aligned}
$$

where we used the representations of $\delta$-function

$$
\begin{aligned}
\delta(x-z) & =\int \frac{d^{4} q}{(2 \pi)^{4}} e^{-i q(x-z)} \\
\delta(x-y) & =\int \frac{d^{4} k}{(2 \pi)^{4}} e^{-i k(x-y)} .
\end{aligned}
$$

The last expression in (2.23) stands for the vacuum polarization tensor. Namely , one can generate the multiple correlation functions of currents $\bar{\psi} T^{a} \gamma^{\mu}\left(\frac{1+\gamma_{5}}{2}\right) \psi$ in the perturbative sense by taking the variational derivative of (2.19) with respect to gauge fields $A_{\mu}^{a}$. This idea also works for the non-gauge currents (2.12) and (2.14). We emphasize that we always take the limit $y=x$ first before the explicit calculation, and thus (2.19) differs from the point-splitting definition of currents.

We now generalize the above definition of currents for the theory defined by (2.5). For this purpose, we rewrite (2.5) as

$$
\mathcal{L}=\bar{\psi} i \mathcal{D} \psi+\bar{\phi} i \mathcal{D}^{\prime} \phi
$$

with

$$
\begin{aligned}
\mathcal{D} & \equiv \not D+i M\left(\frac{1+\gamma_{5}}{2}\right)+i M^{\dagger}\left(\frac{1-\gamma_{5}}{2}\right) \\
\mathcal{D}^{\prime} & \equiv \not D+i M^{\prime}
\end{aligned}
$$

The gauge current (2.10) is then defined by

$$
\begin{aligned}
J^{\mu a}(x) & =\lim _{y \rightarrow x}\left\{<T^{*} \bar{\psi}(y) T^{a} \gamma^{\mu} \psi(x)>+<T^{*} \bar{\phi}(y) T^{a} \gamma^{\mu} \phi(x)>\right\} \\
& =\lim _{y \rightarrow x}\left\{-<T^{*} T^{a} \gamma^{\mu} \psi(x) \bar{\psi}(y)>+<T^{*} T^{a} \gamma^{\mu} \phi(x) \bar{\phi}(y)>\right\} \\
& =\lim _{y \rightarrow x} \operatorname{Tr}\left[T^{a} \gamma^{\mu}\left(\frac{1}{i \mathcal{D}}-\frac{1}{i \mathcal{D}^{\prime}}\right) \delta(x-y)\right]
\end{aligned}
$$


where trace includes the sum over the infinite number of field components in addition to Dirac and Yang-Mills indices. The anti-commuting property of $\psi(x)$ and the commuting property of $\phi(x)$ are used in (2.27).

We next notice the relations

$$
\begin{aligned}
\frac{1}{\mathcal{D}} & =\frac{1}{\mathcal{D}^{\dagger} \mathcal{D}} \mathcal{D}^{\dagger} \\
& =\frac{1}{\not D^{2}+\frac{1}{2} M^{\dagger} M\left(1+\gamma_{5}\right)+\frac{1}{2} M M^{\dagger}\left(1-\gamma_{5}\right)} \mathcal{D}^{\dagger} \\
& =\left[\left(\frac{1+\gamma_{5}}{2}\right) \frac{1}{\not D^{2}+M^{\dagger} M}+\left(\frac{1-\gamma_{5}}{2}\right) \frac{1}{\not D^{2}+M M^{\dagger}}\right] \\
\frac{1}{\mathcal{D}^{\prime}} & =\frac{1}{\left(\mathcal{D}^{\prime}\right)^{\dagger} \mathcal{D}^{\prime}}\left(\mathcal{D}^{\prime}\right)^{\dagger} \\
& =\frac{1}{\not D^{2}+\left(M^{\prime}\right)^{2}}\left(\not D-i M^{\prime}\right) .
\end{aligned}
$$

We thus rewrite $(2.27)$ as

$$
\begin{aligned}
& \operatorname{Tr}\left[-i T^{a} \gamma^{\mu}\left(\frac{1}{\mathcal{D}}-\frac{1}{\mathcal{D}^{\prime}}\right) \delta(x-y)\right] \operatorname{Tr}\left\{-i T^{a} \gamma^{\mu}\left[\left(\frac{1+\gamma_{5}}{2}\right) \sum_{n=0}^{\infty} \frac{1}{\not D^{2}+(2 n \Lambda)^{2}}\right.\right. \\
&+\left(\frac{1-\gamma_{5}}{2}\right) \sum_{n=1}^{\infty} \frac{1}{\not D^{2}+(2 n \Lambda)^{2}} \\
&\left.\left.-\sum_{n=0}^{\infty} \frac{1}{\not D^{2}+[(2 n+1) \Lambda]^{2}}\right] \not D \delta(x-y)\right\} \\
&=\frac{1}{2} \operatorname{Tr}\left[-i T^{a} \gamma^{\mu} \sum_{n=-\infty}^{\infty} \frac{(-1)^{n} \not D^{2}}{\not D^{2}+(n \Lambda)^{2}} \frac{1}{\not D} \delta(x-y)\right] \\
&+\frac{1}{2} \operatorname{Tr}\left[-i T^{a} \gamma^{\mu} \gamma_{5} \frac{1}{\not D} \delta(x-y)\right] \\
&=\frac{1}{2} \operatorname{Tr}\left[T^{a} \gamma^{\mu} f\left(\not D^{2} / \Lambda^{2}\right) \frac{1}{i \not D} \delta(x-y)\right] \\
&+\frac{1}{2} \operatorname{Tr}\left[T^{a} \gamma^{\mu} \gamma_{5} \frac{1}{i \not D} \delta(x-y)\right]
\end{aligned}
$$

where we explicitly evaluated the trace over the infinite number of components and used the fact that the trace over an odd number of $\gamma$-matrices vanishes. We also defined $f\left(x^{2}\right)$ by 


$$
\begin{aligned}
f\left(x^{2}\right) & \equiv \sum_{n=-\infty}^{\infty} \frac{(-1)^{n} x^{2}}{x^{2}+(n \Lambda)^{2}} \\
& =\frac{(\pi x / \Lambda)}{\sinh (\pi x / \Lambda)} .
\end{aligned}
$$

This last expression of (2.30) as a sum of infinite number of terms is given in ref. [1]. The regulator $f\left(x^{2}\right)$, which rapidly approaches 0 at $x^{2}=\infty$, satisfies

$$
\begin{aligned}
f(0) & =1 \\
x^{2} f^{\prime}\left(x^{2}\right) & =0 \text { for } x \rightarrow 0 \\
f(+\infty) & =f^{\prime}(+\infty)=f^{\prime \prime}(+\infty)=\cdots=0 \\
x^{2} f^{\prime}\left(x^{2}\right) & \rightarrow 0 \text { for } x \rightarrow \infty .
\end{aligned}
$$

The essence of the generalized Pauli-Villars regularization (2.5) is thus summarized in terms of regularized currents as follows:

$$
\begin{aligned}
< & \bar{\psi}(x) T^{a} \gamma^{\mu}\left(\frac{1+\gamma_{5}}{2}\right) \psi(x)>_{P V} \\
= & \lim _{y \rightarrow x}\left\{\frac{1}{2} \operatorname{Tr}\left[T^{a} \gamma^{\mu} f\left(\not D^{2} / \Lambda^{2}\right) \frac{1}{i \not D} \delta(x-y)\right]\right. \\
& \left.+\frac{1}{2} \operatorname{Tr}\left[T^{a} \gamma^{\mu} \gamma_{5} \frac{1}{i \not D} \delta(x-y)\right]\right\} \\
<\bar{\psi}(x) \gamma^{\mu}\left(\frac{1+\gamma_{5}}{2}\right) \psi(x)>_{P V} & \lim _{y \rightarrow x}\left\{\frac{1}{2} \operatorname{Tr}\left[\gamma^{\mu} f\left(\not D^{2} / \Lambda^{2}\right) \frac{1}{i \not D} \delta(x-y)\right]\right. \\
& \left.+\frac{1}{2} \operatorname{Tr}\left[\gamma^{\mu} \gamma_{5} \frac{1}{i \not D} \delta(x-y)\right]\right\} \\
<\bar{\psi}(x) \gamma^{\mu} \gamma_{5}\left(\frac{1+\gamma_{5}}{2}\right) \psi(x)>_{P V} & \lim _{y \rightarrow x}\left\{\frac{1}{2} \operatorname{Tr}\left[\gamma^{\mu} \gamma_{5} f\left(\not D^{2} / \Lambda^{2}\right) \frac{1}{i \not D} \delta(x-y)\right]\right. \\
+ & \left.\frac{1}{2} \operatorname{Tr}\left[\gamma^{\mu} \frac{1}{i \not D} \delta(x-y)\right]\right\} .
\end{aligned}
$$

In the left-hand sides of (2.32), the currents are defined in terms of the original fields appearing in (2.1). The axial-vector and vector $U(1)$ currents written in terms of the 
original fields in (2.1) are identical, but the regularized versions (i.e. the last two equations in (2.32)) are different. In particular, the vector $U(1)$ current(i.e. , the second equation in (2.32)) is not completely regularized. See also refs. [2] and [3]. This reflects the different form of naive identities in (2.15) and (2.16) ; if all the currents are well regularized, the naive form of identities would also coincide. We emphasize that all the one-loop diagrams are generated from the (partially) regularized currents in (2.32) ; in other words , (2.32) retains all the information of the generalized Pauli-Villars regularization (2.5).

The trace of energy-momentum tensor generated by the matter field in (2.1) is also interesting. This is related to the variation of field variables 8

$$
\psi(x) \rightarrow e^{-\frac{1}{2} \alpha(x)} \psi(x), \bar{\psi}(x) \rightarrow \bar{\psi}(x) e^{-\frac{1}{2} \alpha(x)}
$$

which is the flat space-time limit of the variation of the weighted variables

$$
\tilde{\psi}(x) \equiv g^{\frac{1}{4}} \psi(x), \tilde{\bar{\psi}}(x) \equiv g^{\frac{1}{4}} \bar{\psi}(x)
$$

under the Weyl transformation

$$
\begin{aligned}
\psi(x) & \rightarrow e^{\frac{3}{2} \alpha(x)} \psi(x) \quad, \bar{\psi}(x) \rightarrow \bar{\psi}(x) e^{\frac{3}{2} \alpha(x)} \\
g_{\mu \nu}(x) & \rightarrow e^{-2 \alpha(x)} g_{\mu \nu}(x) \quad, \quad g=\operatorname{det}_{\mu \nu} .
\end{aligned}
$$

The Noether density generated by the (infinitesimal) transformation (2.33) in (2.1) is given by

$$
\begin{aligned}
\int d^{4} x \bar{\psi}^{\prime}(x) i \not D\left(\frac{1+\gamma_{5}}{2}\right) \psi^{\prime}(x) \\
=-\int d^{4} x \alpha(x) \bar{\psi}(x) \frac{i}{2} \overleftrightarrow{\not D}\left(\frac{1+\gamma_{5}}{2}\right) \psi(x) \\
\quad+\int d^{4} x \bar{\psi}(x) i \not D\left(\frac{1+\gamma_{5}}{2}\right) \psi(x)
\end{aligned}
$$

Following the same procedure as in $(2.32)$, we find 


$$
\begin{aligned}
< & \bar{\psi}(x) \frac{i}{2} \stackrel{\leftrightarrow}{\not D}\left(\frac{1+\gamma_{5}}{2}\right) \psi(x)>_{P V} \\
= & \lim _{y \rightarrow x}\left(\frac{i}{2}\right)\left\{<T^{*} \bar{\psi}(y) \not D_{x}\left(\frac{1+\gamma_{5}}{2}\right) \psi(x)>_{P V}\right. \\
& \left.\quad-<T^{*} \bar{\psi}(y) \overleftarrow{D D}_{y}\left(\frac{1+\gamma_{5}}{2}\right) \psi(x)>_{P V}\right\} \\
= & \lim _{y \rightarrow x}\left(\frac{1}{2}\right) \operatorname{Tr}\left[f\left(\not D^{2} / \Lambda^{2}\right) \delta(x-y)\right] .
\end{aligned}
$$

In summary, eqs $(2.30),(2.32)$ and $(2.37)$ are the basic results of the generalized PauliVillars regularization (2.5).

\section{Covariant Regularization and Covariant Anomalies}

The calculational scheme of covariant anomalies starts with regularized current operators [9] for the theory in (2.1) as follows:

$$
\begin{aligned}
< & \bar{\psi}(x) T^{a} \gamma^{\mu}\left(\frac{1+\gamma_{5}}{2}\right) \psi(x)>_{\operatorname{cov}} \\
= & \lim _{y \rightarrow x} \operatorname{Tr}\left[T^{a} \gamma^{\mu}\left(\frac{1+\gamma_{5}}{2}\right) f\left(\not D^{2} / \Lambda^{2}\right) \frac{1}{i \not D} \delta(x-y)\right] \\
= & \sum_{n} \phi_{n}(x)^{\dagger}\left[T^{a} \gamma^{\mu}\left(\frac{1+\gamma_{5}}{2}\right) f\left(\lambda_{n}^{2} / \Lambda^{2}\right) \frac{1}{i \lambda_{n}}\right] \phi_{n}(x) \\
< & \bar{\psi}(x) \gamma^{\mu}\left(\frac{1+\gamma_{5}}{2}\right) \psi(x)>_{c o v} \\
= & \lim _{y \rightarrow x} \operatorname{Tr}\left[\gamma^{\mu}\left(\frac{1+\gamma_{5}}{2}\right) f\left(\not D^{2} / \Lambda^{2}\right) \frac{1}{i \not D} \delta(x-y)\right] \\
= & \sum_{n} \phi_{n}(x)^{\dagger}\left[\gamma^{\mu}\left(\frac{1+\gamma_{5}}{2}\right) f\left(\lambda_{n}^{2} / \Lambda^{2}\right) \frac{1}{i \lambda_{n}}\right] \phi_{n}(x) \\
< & \bar{\psi}(x) \frac{i}{2} \overleftrightarrow{\not D}\left(\frac{1+\gamma_{5}}{2}\right) \psi(x)>_{\operatorname{cov}} \\
= & \lim _{y \rightarrow x} \operatorname{Tr}\left[\left(\frac{1}{2}\right) f\left(\not D^{2} / \Lambda^{2}\right) \delta(x-y)\right] \\
= & \frac{1}{2} \sum_{n} \phi_{n}(x)^{\dagger} f\left(\lambda_{n}^{2} / \Lambda^{2}\right) \phi_{n}(x)
\end{aligned}
$$

where the complete set $\left\{\phi_{n}(x)\right\}$ is defined by

$$
\not D \phi_{n}(x) \equiv \lambda_{n} \phi_{n}(x)
$$




$$
\begin{aligned}
\int \phi_{m}(x)^{\dagger} \phi_{n}(x) d^{4} x & =\delta_{m, n} \\
\delta_{\alpha \beta} \delta(x-y) & \rightarrow \sum_{n} \phi_{n}(x)_{\alpha} \phi_{n}(y)_{\beta}^{\dagger}
\end{aligned}
$$

with $\alpha$ and $\beta$ including Dirac and Yang-Mills indices.

The function $f\left(x^{2}\right)$ in (3.1) is any smooth function which satisfies the condition (2.31). For the moment, we assume that there is no zero eigenvalue in (3.2). One recognizes a close relation between the generalized Pauli-Villars regularization ( (2.32) and (2.37) ) and the present covariant calculational scheme. The characteristic feature of (3.1) is that it treats the vector and axial-vector components on an equal footing and regularizes them simultaneously. In principle, one could apply different regulator functions, for example , $f\left(\not D^{2} / \Lambda^{2}\right)$ and $g\left(\not D^{2} / \Lambda^{2}\right)$ respectively to vector and axial-vector components in (3.1) instead of using $f\left(\not D^{2} / \Lambda^{2}\right)$ for both of them. In this case , (2.32) is obtained as a special case of (3.1) by taking the limit of either $g\left(\not D^{2} / \Lambda^{2}\right)=1$ or $f\left(\not D^{2} / \Lambda^{2}\right)=1$. In this limit , however, not all the currents are completely regularized.

The anomaly for the current in (3.1) is evaluated as

$$
\begin{aligned}
& D_{\mu}<\bar{\psi}(x) T^{a} \gamma^{\mu}\left(\frac{1+\gamma_{5}}{2}\right) \psi(x)>_{\operatorname{cov}} \\
& \equiv \partial_{\mu}<\bar{\psi}(x) T^{a} \gamma^{\mu}\left(\frac{1+\gamma_{5}}{2}\right) \psi(x)>_{c o v}+g f^{a b c} A_{\mu}^{b}<\bar{\psi}(x) T^{c} \gamma^{\mu}\left(\frac{1+\gamma_{5}}{2}\right) \psi(x)>_{c o v} \\
& =\sum_{n}\left[\phi_{n}(x)^{\dagger} T^{a}\left(\frac{1-\gamma_{5}}{2}\right) f\left(\lambda_{n}^{2} / \Lambda^{2}\right) \frac{1}{i \lambda_{n}}\left(\not D \phi_{n}(x)\right)\right. \\
& \left.-\left(\not D \phi_{n}(x)\right)^{\dagger} T^{a}\left(\frac{1+\gamma_{5}}{2}\right) f\left(\lambda_{n}^{2} / \Lambda^{2}\right) \frac{1}{i \lambda_{n}} \phi_{n}(x)\right] \\
& =\sum_{n}(-i) \phi_{n}(x)^{\dagger} T^{a}\left[\left(\frac{1-\gamma_{5}}{2}\right)-\left(\frac{1+\gamma_{5}}{2}\right)\right] f\left(\lambda_{n}^{2} / \Lambda^{2}\right) \phi_{n}(x) \\
& =i \sum_{n} \phi_{n}(x)^{\dagger} T^{a} \gamma_{5} f\left(\lambda_{n}^{2} / \Lambda^{2}\right) \phi_{n}(x) \\
& =i \sum_{n} \phi_{n}(x)^{\dagger} T^{a} \gamma_{5} f\left(\not D^{2} / \Lambda^{2}\right) \phi_{n}(x) \\
& =i \operatorname{Tr} \int \frac{d^{4} k}{(2 \pi)^{4}} e^{-i k x} T^{a} \gamma_{5} f\left(\not D^{2} / \Lambda^{2}\right) e^{i k x} \\
& =\left(\frac{i g^{2}}{32 \pi^{2}}\right) \operatorname{Tr} T^{a} \epsilon^{\mu \nu \alpha \beta} F_{\mu \nu} F_{\alpha \beta} \quad \text { for } \Lambda \rightarrow \infty
\end{aligned}
$$

where we used the relation (3.2). We also normalized the anti-symmetric symbol as

$$
\epsilon^{1230}=\epsilon^{1234}=1
$$


In the last step of the calculation in (3.3), we replaced the complete set $\left\{\phi_{n}(x)\right\}$ by the plane wave basis for the well-defined operator $f\left(\not D^{2} / \Lambda^{2}\right)$. The final result in (3.3) with $F_{\mu \nu}=\left(\partial_{\mu} A_{\nu}^{a}-\partial_{\nu} A_{\mu}^{a}+g f^{a b c} A_{\mu}^{b} A_{\nu}^{c}\right) T^{a}$ holds for any function $f\left(x^{2}\right)$ which satisfies $(2.31)$. This fact is explained in Appendix for the sake of completeness. Eq.(3.3) shows that only the axial-vector component contributes to the anomaly.

Similarly, the U(1) current in (3.1) satisfies the identity

$$
\begin{aligned}
\partial_{\mu}<\bar{\psi}(x) \gamma^{\mu}\left(\frac{1+\gamma_{5}}{2}\right) \psi(x)>_{c o v} \\
=\sum_{n}\left[-\left(\not D \phi_{n}(x)\right)^{\dagger}\left(\frac{1+\gamma_{5}}{2}\right) f\left(\lambda_{n}^{2} / \Lambda^{2}\right) \frac{1}{i \lambda_{n}} \phi_{n}(x)\right. \\
\left.\quad \quad+\phi_{n}(x)^{\dagger}\left(\frac{1-\gamma_{5}}{2}\right) f\left(\lambda_{n}^{2} / \Lambda^{2}\right) \frac{1}{i \lambda_{n}}\left(\not D \phi_{n}(x)\right)\right] \\
=\quad i \sum_{n} \phi_{n}(x)^{\dagger} \gamma_{5} f\left(\lambda_{n}^{2} / \Lambda^{2}\right) \phi_{n}(x) \\
=i \operatorname{Tr} \int \frac{d^{4} k}{(2 \pi)^{4}} e^{-i k x} \gamma_{5} f\left(\not D^{2} / \Lambda^{2}\right) e^{i k x} \\
=\left(\frac{i g^{2}}{32 \pi^{2}}\right) \operatorname{Tr} \epsilon^{\mu \nu \alpha \beta} F_{\mu \nu} F_{\alpha \beta} \text { for } \Lambda \rightarrow \infty
\end{aligned}
$$

which is the result used in the analysis of baryon number violation [4] and naturally agrees with the result on the basis of the last current in (2.32) in the generalized Pauli-Villars regularization [3]. Again, only the axial component contributes to the anomaly.

The Weyl anomaly in the last relation in (3.1) is evaluated as

$$
\begin{aligned}
<\bar{\psi}(x) \frac{i}{2} \stackrel{\leftrightarrow}{\not D}\left(\frac{1+\gamma_{5}}{2}\right) \psi(x)>_{c o v} & =\frac{1}{2} \operatorname{Tr} \int \frac{d^{4} k}{(2 \pi)^{4}} e^{-i k x} f\left(\not D^{2} / \Lambda^{2}\right) e^{i k x} \\
& =\frac{1}{2}\left(\frac{g^{2}}{24 \pi^{2}}\right) \operatorname{Tr} F^{\mu \nu} F_{\mu \nu} \quad \text { for } \Lambda \rightarrow \infty
\end{aligned}
$$

which is also known to be independent of the choice of $f\left(x^{2}\right)$ in (2.31) 8]. The coefficient of $\operatorname{Tr} F^{\mu \nu} F_{\mu \nu}$ in (3.6) gives the lowest order fermion contribution to the renormalization group $\beta$-function ; $\beta\left(g_{r}\right)=\left(\frac{1}{2}\right) g_{r}^{3} /\left(24 \pi^{2}\right)$.

The anomaly in (3.3) is covariant under gauge transformation, which is the reason why (3.3) is called "covariant anomaly" [10. From the diagramatic view point, all the vertices of one-loop diagrams are regularized by the gauge invariant regulator $f\left(\lambda_{n}^{2} / \Lambda^{2}\right)$ except 
for the vertex corresponding to the Noether current itself. Because of this asymmetric treatment of vertices, the anomaly (3.3) does not satisfy the so-called integrability (or Wess-Zumino consistency) condition [11]. The relation (3.3) however specifies precisely the essence of the anomaly, namely, one cannot impose gauge invariance on all the vertices of anomalous diagrams. It is also known that one can readily convert the covariant anomaly in (3. 3) to the anomaly which satisfies the Wess-Zumino condition [10].

The anomaly in (3.3) vanishes for

$$
\operatorname{Tr} T^{a}\left\{T^{b}, T^{c}\right\}=0
$$

For the anomaly-free gauge theory, which satisfies (3.7), one can impose gauge invariance on all the gauge vertices, and consequently the Bose symmetry is recovered. The regularization (3.1) thus provides a natural regularization of all the one-loop diagrams in anomaly-free gauge theory.

From the analysis presented above, one can understand the consistency of the "partial regularization" of the generalized Pauli-Villars regularization (2.32) and (2.37) for anomaly-free gauge theory, except for the second expression in (2.32) which cannot produce $\mathrm{U}(1)$ anomaly by the naive treatment of the right-hand side. Our analysis, which is based on well-regularized operators in (3.1), provides a transparent way to understand the conclusions in refs. [1], [目] and [3].

We now add several comments on the covariant regularization (3.1). First of all, the regularization (3.1) should not be confused with the higher derivative regularization, for example ,

$$
\mathcal{L}=\bar{\psi}(x) i \not D\left(\frac{\not D^{2}+\Lambda^{2}}{\Lambda^{2}}\right)^{2}\left(\frac{1+\gamma_{5}}{2}\right) \psi(x) .
$$

*In the generalized Pauli-Villars regularization, all the vertices are treated on an equal footing and Bose symmetrically since the regularization is implemented in the Lagrangian level. The evaluation of anomaly for gauge couplings, if it should be performed in the generalized Pauli-Villars regularization , would therefore be quite different from the anomaly calculation in the covariant regularization (3.1). The axial-vector component of gauge current, which could produce gauge anomaly, is however not regularized in (2.5), as is seen in (2.32). The regularized gauge current satisfies the naive identity (2.15) and, consequently, the regularization (2.5) as it stands is not applicable to the evaluation of possible gauge anomaly. 
If one chooses $f\left(x^{2}\right) \equiv\left[\Lambda^{2} /\left(x^{2}+\Lambda^{2}\right)\right]^{2}$ in (3.1), the regularization (3.1) resembles the theory defined by (3.8). However, this resemblance is spurious, since the Noether current given by (3.8) contains higher derivative terms in addition to the minimal one and thus the one-loop diagrams are $\underline{\text { not }}$ regularized by (3.8). It is an interesting question whether (3.1) for anomaly-free gauge theory can be implemented in the Lagrangian level if one incorporates an infinite number of regulator fields.

Secondly, the regularization (3.1) can be implemented for more general theory such as

$$
\begin{aligned}
\mathcal{L} & =\bar{\psi} i \gamma^{\mu}\left[\partial_{\mu}-i R_{\mu}^{a}(x) T^{a}\left(\frac{1+\gamma_{5}}{2}\right)-i L_{\mu}^{a}(x) T^{a}\left(\frac{1-\gamma_{5}}{2}\right)\right] \psi \\
& =\bar{\psi} i \gamma^{\mu}\left(\partial_{\mu}-i R_{\mu}\right)\left(\frac{1+\gamma_{5}}{2}\right) \psi+\bar{\psi} i \gamma^{\mu}\left(\partial_{\mu}-i L_{\mu}\right)\left(\frac{1-\gamma_{5}}{2}\right) \psi
\end{aligned}
$$

In this case, left-and right-handed components separately satisfy the identities [5, 9]

$$
D_{\mu}<\bar{\psi} T^{a} \gamma^{\mu}\left(\frac{1 \pm \gamma 5}{2}\right) \psi>_{c o v}= \pm\left(\frac{i}{32 \pi^{2}}\right) \operatorname{Tr} T^{a} \epsilon^{\mu \nu \alpha \beta} F_{\mu \nu} F_{\alpha \beta}
$$

The covariant regularization is quite flexible and works for arbitrary gauge theory ; for example, one can readily show that Yukawa couplings do not modify anomaly [9].

Finally, we comment on the treatment of zero modes of $\not D$ in (3.1) in some detail ; this problem is also shared by the generalized Pauli-Villars regularization (2.32) in non-perturbative analysis. When the gauge field $A_{\mu}(x)$ is topologically non-trivial, the eigenvalue equation (3.2) contains well-defined zero eigenvalues [12]. The definition of current operators thus becomes subtle, but the divergence of currents such as (3.3) and (3.5) does not contain the singular factor $1 / \lambda_{n}$ and thus anomalies themselves are welldefined. In the path integral framework, this situation is treated in the following way:

One first notices that $\gamma_{5} \phi_{n}(x)$ belongs to the eigenvalue $-\lambda_{n}$ in $(3.2)$ since $\left\{\gamma_{5}, \not D\right\}=0$. We thus define new complete basis sets [6]

$$
\begin{aligned}
\phi_{n}^{R}(x) & \equiv\left(\frac{1+\gamma_{5}}{\sqrt{2}}\right) \phi_{n}(x) \text { if } \lambda_{n}>0 \\
& \equiv\left(\frac{1+\gamma_{5}}{2}\right) \phi_{n}(x) \text { if } \lambda_{n}=0 \\
\phi_{n}^{L}(x) & \equiv\left(\frac{1-\gamma_{5}}{\sqrt{2}}\right) \phi_{n}(x) \text { if } \lambda_{n}>0
\end{aligned}
$$




$$
\equiv\left(\frac{1-\gamma_{5}}{2}\right) \phi_{n}(x) \text { if } \lambda_{n}=0
$$

Note that $\phi_{n}(x)$ with $\lambda_{n}=0$ can be chosen to be the eigenvector of $\gamma_{5}$. We thus expand

$$
\begin{aligned}
\psi_{R}(x) & =\left(\frac{1+\gamma_{5}}{2}\right) \psi(x) \\
& =\sum_{\lambda_{n} \geq 0} a_{n} \phi_{n}^{R}(x) \\
\bar{\psi}_{R}(x) & =\sum_{\lambda_{n} \geq 0} \overline{b_{n}} \phi_{n}^{L}(x)^{\dagger}
\end{aligned}
$$

where $a_{n}$ and $\overline{b_{n}}$ are Grassmann numbers, and the action (2.1) and the path integral measure are formally defined by

$$
\begin{aligned}
S & =\int \mathcal{L} d^{4} x=\sum_{\lambda_{n}>0} \lambda_{n} \overline{b_{n}} a_{n} \\
d \mu & =\mathcal{D} \bar{\psi}_{R} \mathcal{D} \psi_{R}=\prod_{\lambda_{n} \geq 0} d \overline{b_{n}} d a_{n}
\end{aligned}
$$

The Jacobian factor under the (infinitesimal) chiral U(1) transformation

$$
\begin{aligned}
\psi_{R}^{\prime}(x) & =e^{i \alpha(x) \gamma_{5}} \psi_{R}(x)=e^{i \alpha(x)} \psi_{R}(x) \\
\bar{\psi}_{R}^{\prime}(x) & =\bar{\psi}_{R}(x) e^{-i \alpha(x)}
\end{aligned}
$$

is given by

$$
\begin{aligned}
J & =\exp \left[-i \int d^{4} x \alpha(x) \sum_{\lambda_{n} \geq 0}\left\{\phi_{n}^{R}(x)^{\dagger} \phi_{n}^{R}(x)-\phi_{n}^{L}(x)^{\dagger} \phi_{n}^{L}(x)\right\}\right] \\
& =\exp \left[-i \int d^{4} x \alpha(x) \sum_{\text {all } \lambda_{n}} \phi_{n}^{\dagger}(x) \gamma_{5} \phi_{n}(x)\right]
\end{aligned}
$$

The sum of terms in (3.15) may be defined by

$$
\lim _{N \rightarrow \infty} \sum_{n=1}^{N} \phi_{n}(x)^{\dagger} \gamma_{5} \phi_{n}(x)=\lim _{\Lambda \rightarrow \infty} \sum_{n=1}^{\infty} \phi_{n}(x)^{\dagger} \gamma_{5} f\left(\lambda_{n}^{2} / \Lambda^{2}\right) \phi_{n}(x)
$$

by replacing the mode cut-off, which is natural for the definition (3.13), by the cut-off in $\lambda_{n}$ by using a suitable regulator satisfying (2.31). By this way, one directly obtains the anomaly factor (3.5) as a Jacobian without referring to current operators. In the 
generalized Pauli-Villars regularization (2.17) the measure is shown to be invariant under the chiral $\mathrm{U}(1)$ transformation but the variation of action, which corresponds to the right-hand side of (2.16), gives rise to the same anomaly factor as in (3.5) for $\Lambda \rightarrow \infty$.

By recalling that $\gamma_{5} \phi_{n}(x)$ belongs to the eigenvalue $-\lambda_{n}$ in (3.2), the integration of (3.5) gives rise to

$$
\begin{aligned}
n_{+}-n_{-} & =\sum_{n} \int \phi_{n}(x)^{\dagger} \gamma_{5} f\left(\lambda_{n}^{2} / \Lambda^{2}\right) \phi_{n}(x) d^{4} x \\
& =\frac{g^{2}}{32 \pi^{2}} \int \operatorname{Tr} \epsilon^{\mu \nu \alpha \beta} F_{\mu \nu} F_{\alpha \beta} d^{4} x \\
& =\nu
\end{aligned}
$$

where $n_{ \pm}$stand for the number of zero modes with $\gamma_{5}= \pm 1$, and $\nu$ is the Pontryagin index (or instanton number) [12]. Note that the replacement (3.16) is consistent with (3.17). From (3.15) and (3.17), we obtain

$$
d \mu \rightarrow d \mu \exp [-i \alpha \nu]
$$

for the x-independent $\alpha$ in (3.14). In (2.17), the variation of the action gives rise to the same phase factor as (3.18) for $\Lambda \rightarrow \infty$. Eqs.(3.14) and (3.18) show that

$$
<\psi_{R}(x) \cdots \bar{\psi}_{R}(y) \cdots>=\int d \mu\left[\psi_{R}(x) \cdots \bar{\psi}_{R}(y) \cdots\right] \exp \left[\int \mathcal{L} d^{4} x\right]
$$

is non-vanishing only for the Green's functions which contain $\nu$ more $\psi$ variables than $\bar{\psi}$ variables. This gives the chirality selection rule and the fermion number non- conservation. Since the zero modes do not appear in the action (3.13), the path integral (i.e., leftderivative) over Grassmann variables corresponding to zero modes is completely consumed by $n_{+} \psi$-variables and $n_{-} \bar{\psi}$-variables appearing in Green's functions. As a result, the Green's function and current operators do not contain the (singular) inverse of zero eigenvalues any more, which could arise from the action. This procedure is thus consistent for anomaly-free gauge theory.

\footnotetext{
*As for the vector-like transformation corresponding to the naive identities (2.15), the Jacobian is shown to be non-vanishing and give rise to (3.3) and (3.5) ; this evaluation of Jacobian corresponds to super-imposing the covariant regularization on the generalized Pauli-Villars regularization (2.5)
} 
When the gauge group contains anomaly, the Jacobian for the gauge transformation (2.8) with gauge field kept fixed gives the covariant anomaly (3.3) for the Noether current. In this case, the variation of the partition function under the change of path integral variables has a definite meaning, but the partition function itself is ill-defined since the anomalous gauge theory cannot be completely regularized by gauge invariant cut-off in terms of $\lambda_{n}$ : To define the partition function, one needs to use a regulator which explicitly breaks gauge invariance such as the conventional Pauli-Villars regularization [13].

\section{Covariant Regularization of Anomaly-free Theory}

In view of the partial regularization (2.32) of the generalized Pauli- Villars regularization, it is interesting to apply the fully regularized expressions (3.1) for the practical calculations in anomaly- free gauge theory. We then enjoy much more freedom in choosing the regulator $f\left(x^{2}\right)$, simply because we do not require a Lagrangian-level implementation of $f\left(x^{2}\right)$. We illustrate this application by evaluating the vacuum polarization tensor. To be specific, we evaluate the vacuum polarization tensor for QED (in Euclidean metric)

$$
\mathcal{L}=\bar{\psi} i \gamma^{\mu}\left(\partial_{\mu}-i e A_{\mu}\right) \psi-m \bar{\psi} \psi
$$

by choosing a simple regulator

$$
f\left(x^{2}\right)=\left(\frac{\Lambda^{2}}{x^{2}+\Lambda^{2}}\right)^{2}
$$

which is convenient for practical calculations and satisfies the condition (2.31). The final result can be readily extended to the chiral theory defined by the first current in (3.1).

We thus start with a regularized current

$$
<\bar{\psi}(x) \gamma^{\mu} \psi(x)>_{\operatorname{cov}}=\lim _{y \rightarrow x} \operatorname{Tr}\left\{\gamma^{\mu} \frac{1}{i \not D-m}\left(\frac{\Lambda^{2}}{\not D^{2}+\Lambda^{2}}\right)^{2} \delta(x-y)\right\}
$$

with

$$
\not D=\gamma^{\mu}\left(\partial_{\mu}-i e A_{\mu}\right)
$$

By expanding (4.3) in powers of $e A_{\mu}$ and retaining only the terms linear in $e A_{\mu}$, one obtains

$$
<\bar{\psi}(x) \gamma^{\mu} \psi(x)>_{\text {cov }}
$$




$$
\begin{array}{rl}
=\lim _{y \rightarrow x} & T r\left\{\gamma^{\mu}\left[\frac{1}{i \not \partial-m}(-e \not A) \frac{1}{i \not \partial-m}\left(\frac{\Lambda^{2}}{\not \partial^{2}+\Lambda^{2}}\right)^{2} \delta(x-y)\right]\right. \\
+\gamma^{\mu} & \frac{1}{i \not \partial-m}\left(\frac{\Lambda^{2}}{\not \partial^{2}+\Lambda^{2}}\right)^{2}(i e)\left\{\left(\partial_{\nu} A^{\nu}\right)+2 A^{\nu} \partial_{\nu}+\frac{1}{4}\left[\gamma^{\alpha}, \gamma^{\nu}\right] F_{\alpha \nu}\right\} \\
& \times\left(\frac{1}{\not \partial^{2}+\Lambda^{2}}\right) \delta(x-y) \\
+\gamma^{\mu} & \frac{1}{i \not \partial-m}\left(\frac{1}{\not \supset^{2}+\Lambda^{2}}\right)(i e)\left\{\left(\partial_{\nu} A^{\nu}\right)+2 A^{\nu} \partial_{\nu}+\frac{1}{4}\left[\gamma^{\alpha}, \gamma^{\nu}\right] F_{\alpha \nu}\right\} \\
& \left.\times\left(\frac{\Lambda^{2}}{\not \partial^{2}+\Lambda^{2}}\right)^{2} \delta(x-y)\right\}
\end{array}
$$

where we used

$$
\begin{aligned}
\not D^{2} & =\frac{1}{2}\left\{\gamma^{\mu}, \gamma^{\nu}\right\} D_{\mu} D_{\nu}+\frac{1}{2}\left[\gamma^{\mu}, \gamma^{\nu}\right] D_{\mu} D_{\nu} \\
& =D_{\mu} D^{\mu}-\frac{i e}{4}\left[\gamma^{\mu}, \gamma^{\nu}\right] F_{\mu \nu} \\
& =\partial_{\mu} \partial^{\mu}-i e\left[\partial_{\mu} A^{\mu}+A^{\mu} \partial_{\mu}\right]-e^{2} A_{\mu} A^{\mu}-\frac{i e}{4}\left[\gamma^{\mu}, \gamma^{\nu}\right] F_{\mu \nu} \\
& =\partial_{\mu} \partial^{\mu}-i e\left[\left(\partial_{\mu} A^{\mu}\right)+2 A^{\mu} \partial_{\mu}\right]-e^{2} A_{\mu} A^{\mu}-\frac{i e}{4}\left[\gamma^{\mu}, \gamma^{\nu}\right] F_{\mu \nu}
\end{aligned}
$$

with $F_{\mu \nu}=\partial_{\mu} A_{\nu}-\partial_{\nu} A_{\mu}$. The derivative operator $\partial_{\mu}$ in (4.5) and (4.6), except the one in $\left(\partial_{\mu} A^{\mu}\right)$ and $F_{\mu \nu}$, acts on all the x-variables standing on the right of it. By taking the variational derivative of (4.5) with respect to $e A_{\nu}(z)$, one finds a regularized expression of the vacuum polarization tensor

$$
\begin{aligned}
\lim _{y \rightarrow x} & \operatorname{Tr}\left\{\gamma^{\mu} \frac{(-1)}{i \not \partial-m} \gamma^{\nu} \delta(x-z)\left(\frac{\Lambda^{2}}{\not^{2}+\Lambda^{2}}\right)^{2} \delta(x-y)\right. \\
& +\gamma^{\mu} \frac{1}{i \not \partial-m}\left(\frac{\Lambda^{2}}{\not^{2}+\Lambda^{2}}\right)^{2}(i)\left[\left(\partial^{\nu} \delta(x-z)\right)+2 \delta(x-z) \partial^{\nu}\right. \\
& \left.+\frac{1}{2}\left[\gamma^{\alpha}, \gamma^{\nu}\right]\left(\partial_{\alpha} \delta(x-z)\right)\right] \times\left(\frac{1}{\not^{2}+\Lambda^{2}}\right) \delta(x-y) \\
& +\gamma^{\mu} \frac{1}{i \not \partial-m}\left(\frac{1}{\not \partial^{2}+\Lambda^{2}}\right)(i)\left[\left(\partial^{\nu} \delta(x-z)\right)+2 \delta(x-z) \partial^{\nu}\right. \\
& \left.\left.+\frac{1}{2}\left[\gamma^{\alpha}, \gamma^{\nu}\right]\left(\partial_{\alpha} \delta(x-z)\right)\right] \times\left(\frac{\Lambda^{2}}{\not \partial^{2}+\Lambda^{2}}\right)^{2} \delta(x-y)\right\}
\end{aligned}
$$


We now use (2.24) in (4.7), and we obtain the momentum representation of the vacuum polarization tensor (see also (2.23))

$$
\begin{aligned}
\Pi^{\mu \nu}(q)= & \int \frac{d^{4} k}{(2 \pi)^{4}} \operatorname{Tr}\left\{\gamma^{\mu} \frac{(-1)}{\not k+\not 1-m} \gamma^{\nu} \frac{1}{\not k-m}\left(\frac{\Lambda^{2}}{-k^{2}+\Lambda^{2}}\right)^{2}\right. \\
+ & +\gamma^{\mu} \frac{1}{\not k+\not \subset-m}\left(\frac{\Lambda^{2}}{-(k+q)^{2}+\Lambda^{2}}\right)^{2}\left[q^{\nu}+2 k^{\nu}+\frac{1}{2}\left[\gamma^{\alpha}, \gamma^{\nu}\right] q_{\alpha}\right] \\
& \times\left(\frac{1}{-k^{2}+\Lambda^{2}}\right) \\
+ & \gamma^{\mu} \frac{1}{\not k+\not h-m}\left(\frac{1}{-(k+q)^{2}+\Lambda^{2}}\right)\left[q^{\nu}+2 k^{\nu}+\frac{1}{2}\left[\gamma^{\alpha}, \gamma^{\nu}\right] q_{\alpha}\right] \\
& \left.\times\left(\frac{\Lambda^{2}}{-k^{2}+\Lambda^{2}}\right)^{2}\right\} .
\end{aligned}
$$

The first term in (4.8) stands for the naive momentum cut-off by a form factor, which generally spoils gauge invariance. The remaining two terms in (4.8) recover gauge invariance spoiled by the first term.

After the standard trace calculation and using the Feynman parameters, the first term in (4.8) gives

$$
\begin{aligned}
\left(\frac{\Lambda^{4}}{4 \pi^{2}}\right) \int_{0}^{1} d \alpha & \int_{0}^{1-\alpha} d \beta\left\{-g^{\mu \nu} \frac{\beta}{-\alpha(1-\alpha) q^{2}+(1-\beta) m^{2}+\beta \Lambda^{2}}\right. \\
+ & {\left[-m^{2} g^{\mu \nu}-\alpha(1-\alpha) g^{\mu \nu} q^{2}+2 \alpha(1-\alpha) q^{\mu} q^{\nu}\right] } \\
& \left.\times \frac{\beta}{\left[-\alpha(1-\alpha) q^{2}+(1-\beta) m^{2}+\beta \Lambda^{2}\right]^{2}}\right\} \\
\rightarrow \quad\left(\frac{1}{4 \pi^{2}}\right) & \int_{0}^{1} d \alpha 2 \alpha(1-\alpha)\left(q^{\mu} q^{\nu}-g^{\mu \nu} q^{2}\right) \ln \left[\frac{\Lambda^{2}}{-\alpha(1-\alpha) q^{2}+m^{2}}\right] \\
+ & \left(\frac{1}{4 \pi^{2}}\right)\left[\frac{-1}{2}\left(\Lambda^{2}-m^{2}\right) g^{\mu \nu}+\frac{1}{6} g^{\mu \nu} q^{2}\right. \\
& \left.-\frac{1}{3} q^{\mu} q^{\nu}-\frac{5}{18}\left(q^{\mu} q^{\nu}-g^{\mu \nu} q^{2}\right)\right]
\end{aligned}
$$

for $\Lambda \rightarrow$ large. Similarly, the second and third terms in (4.8) together give

$$
\begin{aligned}
\left(\frac{\Lambda^{4}}{4 \pi^{2}}\right) \int_{0}^{1} d & \alpha \int_{0}^{1-\alpha} d \beta\left\{g^{\mu \nu} \frac{1-\beta}{-\alpha(1-\alpha) q^{2}+\beta m^{2}+(1-\beta) \Lambda^{2}}\right. \\
& -\left[\alpha(2 \alpha-1) q^{\mu} q^{\nu}+\alpha\left(g^{\mu \nu} q^{2}-q^{\mu} q^{\nu}\right)\right]
\end{aligned}
$$




$$
\begin{gathered}
\left.\times \frac{1-\beta}{\left[-\alpha(1-\alpha) q^{2}+\beta m^{2}+(1-\beta) \Lambda^{2}\right]^{2}}\right\} \\
\rightarrow\left(\frac{1}{4 \pi^{2}}\right)\left[\frac{1}{2}\left(\Lambda^{2}-m^{2}\right) g^{\mu \nu}+\frac{5}{36} g^{\mu \nu} q^{2}\right. \\
\left.+\frac{1}{36} q^{\mu} q^{\nu}+\frac{1}{4}\left(q^{\mu} q^{\nu}-g^{\mu \nu} q^{2}\right)\right]
\end{gathered}
$$

for $\Lambda \rightarrow$ large. These two expressions in (4.9) and (4.10) put together finally give rise to the familiar gauge invariant result

$$
\left(\frac{1}{4 \pi^{2}}\right)\left(q^{\mu} q^{\nu}-g^{\mu \nu} q^{2}\right)\left\{\int_{0}^{1} d \alpha 2 \alpha(1-\alpha) \ln \left[\frac{\Lambda^{2}}{-\alpha(1-\alpha) q^{2}+m^{2}}\right]-\frac{1}{3}\right\} .
$$

The result for the chiral gauge theory (2.1) is obtained from (4.11) by setting $m=0$ and multiplying it by $\frac{1}{2} \operatorname{Tr} T^{a} T^{b}$.

The covariant regularization scheme thus gives rise to a gauge invariant result on the basis of well-regularized finite calculations. It is important that we always stay in $\mathrm{d}=4$ dimensional space-time in this calculation. This property is crucial for a reliable treatment of the anomaly. The coefficient of $\ln \Lambda^{2}$ in (4.11), which is related to the renormalization group $\beta$-function, is independent of the choice of $f\left(x^{2}\right)$ in $(4.2)$. For example, one can confirm that

$$
f\left(x^{2}\right)=\left(\frac{\Lambda^{2}}{x^{2}+\Lambda^{2}}\right)^{n}, n \geq 2
$$

gives the same numerical coefficient of $\ln \Lambda^{2}$ by dividing (4.9) and (4.10) by $\Lambda^{4}$ and taking suitable derivatives with respect to $\Lambda^{2}$. The finite term, $-1 / 3$, in (4.11) depends on the specific regulator ; this is not a drawback since the finite term is uniquely fixed by the renormalization condition in renormalizable theory. The regulator independence of the coefficient of $\ln \Lambda^{2}$ is also expected from the fact that the Weyl anomaly in (3.6) (and related $\beta$-function)is independent of $f\left(x^{2}\right)$.

The present covariant regularization can be readily applied to the calculations of higher point functions and to practical calculations in chiral gauge theory such as the WeinbergSalam theory ; the covariant regularization can handle gauge anomalies in a reliable way, and thus one can treat lepton and quark sectors separately without grouping the fermions into a multiplet of $\mathrm{SO}(10)$. The Higgs coupling, which mixes left-and righthanded components, is readily handled by the present method as is explained in [9]. 


\section{Discussion and Conclusion}

Motivated by the interesting suggestion of generalized Pauli-Villars regularization, we reexamined the regularization and anomalies in gauge theory. The generalized Pauli-Villars regularization as reformulated as a regularization of component operators in this paper will perhaps make the covariant regularization, which has been known for some time, more acceptable ; the Lagrangian level realization of the covariant regularization for anomalyfree gauge theory however remains as an open question. The covariant regularization spoils the Bose symmetry for anomalous gauge theory, but it preserves the Bose symmetry as well as gauge invariance for anomaly-free gauge theory.

Our analysis here is confined to one-loop level calculations, though certain nonperturbative aspects such as instantons are also involved. As for multi-loop diagrams, the higher derivative regularization in the sector of gauge fields [14], for example, can render all the multi-loop diagrams finite. The one-loop diagrams which include only the gauge fields cannot be regularized by the higher derivative regularization but they can be covariantly regularized if one uses the covariant background gauge technique 15. In fact, we recently illustrated a simple non-diagramatic calculation of one-loop $\beta$-function of QCD by using the method of covariant anomaly 16].

The generalized Pauli-Villars regularization is also known to have interesting implications on lattice gauge theory [17], but its analysis is beyond the scope of the present paper.

In conclusion, we have shown that the basic mechanism of generalized Pauli-Villars regularization of continuum theory is made transparent if one looks at it from the view point of a regularization of composite current operators ; by this way, one can readily

compare the generalized Pauli-Villars regularization with the covariant regularization of chiral gauge theory. The covariant regularization scheme, which is quite flexible, has been also shown to be useful in practical calculations. 


\section{Appendix}

For the sake of completeness, we here quote the proof of $\mathrm{f}(\mathrm{x})$ - independence of (3.3) and (3.5)[6]. The calculation of (3.5), for example, proceeds as

$$
\begin{aligned}
& i \operatorname{Tr} \int \frac{d^{4} k}{(2 \pi)^{4}} e^{-i k x} \gamma_{5} f\left(\not D^{2} / \Lambda^{2}\right) e^{i k x} \\
& =i \operatorname{Tr} \int \frac{d^{4} k}{(2 \pi)^{4}} \gamma_{5} f\left(\frac{\left(i k_{\mu}+D_{\mu}\right)\left(i k^{\mu}+D^{\mu}\right)-\frac{i g}{4}\left[\gamma^{\mu}, \gamma^{\nu}\right] F_{\mu \nu}}{\Lambda^{2}}\right) \\
& =i \Lambda^{4} \operatorname{Tr} \int \frac{d^{4} k}{(2 \pi)^{4}} \gamma_{5} f\left(-k_{\mu} k^{\mu}+\frac{2 i k^{\mu} D_{\mu}}{\Lambda}+\frac{D^{\mu} D_{\mu}}{\Lambda^{2}}\right. \\
& \left.\quad-\frac{i g}{4 \Lambda^{2}}\left[\gamma^{\mu}, \gamma^{\nu}\right] F_{\mu \nu}\right)
\end{aligned}
$$

where we used

$$
\begin{aligned}
\not D^{2} & =\frac{1}{2}\left\{\gamma^{\mu}, \gamma^{\nu}\right\} D_{\mu} D_{\nu}+\frac{1}{2}\left[\gamma^{\mu}, \gamma^{\nu}\right] D_{\mu} D_{\nu} \\
& =D_{\mu} D^{\mu}-\frac{i g}{4}\left[\gamma^{\mu}, \gamma^{\nu}\right] F_{\mu \nu}
\end{aligned}
$$

and re-scaled the variable $k_{\mu} \rightarrow \Lambda k_{\mu}$. We next expand the quantity involving $\mathrm{f}(\mathrm{x})$ in (A.1) around $x=-k_{\mu} k^{\mu}=\left|k^{2}\right|$ as

$$
\begin{aligned}
f\left(-k_{\mu} k^{\mu}+\frac{2 i k^{\mu} D_{\mu}}{\Lambda}+\frac{D^{\mu} D_{\mu}}{\Lambda^{2}}-\frac{i g}{4 \Lambda^{2}}\left[\gamma^{\mu}, \gamma^{\nu}\right] F_{\mu \nu}\right) \\
=f\left(-k_{\mu} k^{\mu}\right)+f^{\prime}\left(-k_{\mu} k^{\mu}\right)\left\{\frac{2 i k^{\mu} D_{\mu}}{\Lambda}+\frac{D^{\mu} D_{\mu}}{\Lambda^{2}}-\frac{i g}{4 \Lambda^{2}}\left[\gamma^{\mu}, \gamma^{\nu}\right] F_{\mu \nu}\right\} \\
\quad+\frac{1}{2 !} f^{\prime \prime}\left(-k_{\mu} k^{\mu}\right)\left\{\frac{2 i k^{\mu} D_{\mu}}{\Lambda}+\frac{D^{\mu} D_{\mu}}{\Lambda^{2}}-\frac{i g}{4 \Lambda^{2}}\left[\gamma^{\mu}, \gamma^{\nu}\right] F_{\mu \nu}\right\}^{2}+\ldots
\end{aligned}
$$

When $\Lambda \rightarrow \infty$, only the terms of order $1 / \Lambda^{4}$ or larger in (A.3) survive in (A.1). Moreover, the trace $\operatorname{Tr}\left(\gamma_{5} \ldots\right)$ is non-vanishing only for the terms with more than four $\gamma$-matrices. The only term that satisfies these two conditions is the third term in (A. 3) with $\left(\left[\gamma^{\mu}, \gamma^{\nu}\right] F_{\mu \nu}\right)^{2}$. The calculation of (A.1) thus becomes

$$
i \operatorname{Tr} \int \frac{d^{4} k}{(2 \pi)^{4}} \gamma_{5} \frac{1}{2 !} f^{\prime \prime}\left(-k_{\mu} k^{\mu}\right)\left\{\frac{-i g}{4}\left[\gamma^{\mu}, \gamma^{\nu}\right] F_{\mu \nu}\right\}^{2}
$$




$$
\begin{aligned}
& =i \operatorname{Tr} \gamma_{5} \frac{1}{2}\left\{\frac{-i g}{4}\left[\gamma^{\mu}, \gamma^{\nu}\right] F_{\mu \nu}\right\}^{2} \frac{1}{16 \pi^{2}} \int_{0}^{\infty} d x x f^{\prime \prime}(x) \\
& =\left(\frac{i g^{2}}{32 \pi^{2}}\right) \operatorname{Tr} \epsilon^{\mu \nu \alpha \beta} F_{\mu \nu} F_{\alpha \beta}
\end{aligned}
$$

after taking the trace over $\gamma$-matrices: We here used $d^{4} k=\pi^{2}\left|k^{2}\right| d\left|k^{2}\right|$, and

$$
\begin{aligned}
\int_{0}^{\infty} d x x f^{\prime \prime}(x) & =\left.x f^{\prime}(x)\right|_{0} ^{\infty}-\int_{0}^{\infty} d x f^{\prime}(x) \\
& =-\left.f(x)\right|_{0} ^{\infty}=f(0)=1
\end{aligned}
$$

by noting the conditions (2.31) including $x f^{\prime}(x) \rightarrow 0$ for $x \rightarrow \infty$. The result (A.4) is thus independent of the regulator $\mathrm{f}(\mathrm{x})$; the convenient choice of $\mathrm{f}(\mathrm{x})$ for practical calculations is $(4.12)$ or $f(x)=\exp [-x]$.

The above analysis is also applicable to (3.3). It is known that a similar analysis holds for $(3.6)[8]$.

\section{Note Added}

After submitting the present paper, the works by Narayanan and Neuberger [18] on the Kaplan's formulation came to my attention. These authors analyze two-dimensional gauge anomaly , in particular consistent from of anomaly, from a view point of $2+1$ dimensional theory. This calculational scheme is apparently different from the generalized Pauli-Villars regularization in (2.5) , which is not applicable to the evaluation of gauge anomaly.

\section{References}

[1] S.A.Frolov and A.A.Slavnov, Phys. Lett. B309 (1993)344

[2] R.Narayanan and H.Neuberger, Phys. Lett.B301(1993)62

[3] S.Aoki and Y.Kikukawa, Mod. Phys. Lett. A8(1993)3517

[4] G.'t Hooft, Phys. Rev. Lett. 37(1976)8 ;

Phys. Rev. D14(1976)172 ; D18(1978)2199(E)

[5] S.Adler, Phys.Rev. 177(1969)2426

J.S.Bell and R.Jackiw, Nuovo Cim, A60(1969)47 
[6] K.Fujikawa, Phys. Rev. D21(1980)2848; 22(1980)1499(E);

Phys. Rev. Lett. 42(1979)1195

[7] L.Alvarez-Gaumé and E.Witten, Nucl. Phys. B234(1983)269

[8] K.Fujikawa, Phys. Rev. Lett. 44(1980)1733;

Phys. Rev. D23(1981)2262

[9] K.Fujikawa, Phys. Rev. D29(1984)285

[10] W.A.Bardeen and B.Zumino, Nucl. Phys. B244(1984)421

[11] J.Wess and B.Zumino, Phys. Lett. 37B(1971)95

[12] M.Atiyah and I.Singer, Ann. Math. 87(1968)484

[13] W.A.Bardeen, Phys. Rev. 184(1969)1848

H.Shinke and H.Suzuki, Mod. Phys. Lett. A40(1993)3835

[14] L.D.Faddeev and A.A.Slavnov, GaugeFields (Benjamin / Cummings, 1980)

[15] G.'t Hooft, Nucl. Phys. B62(1973)444

[16] K.Fujikawa, Phys. Rev. D48(1993)3922

[17] D.B.Kaplan, Phys. Lett. B288(1992)342

[18] R.Narayanan and H.Neuberger , Phys. Rev. Lett. 71(1993)3251 ;

Nucl. Phys. B412(1994)574 